\title{
Gangrena de Fournier: estudio de los factores pronósticos en 90 pacientes
}

\author{
Medina Polo J, Tejido Sánchez A, de la Rosa Kehrmann F, Felip Santamaría N, \\ Blanco Álvarez M, Leiva Galvis O.
}

Servicio de Urología. Hospital Universitario 12 de Octubre. Madrid.

\author{
Actas Urol Esp. 2008;32(10):1024-1030
}

\begin{abstract}
RESUMEN
GANGRENA DE FOURNIER: ESTUDIO DE LOS FACTORES PRONÓSTICOS EN 90 PACIENTES

Introducción: Este estudio evalúa los factores de riesgo y aquellas variables pronósticas que influyen en la supervivencia de los pacientes con gangrena de Fournier.

Material y métodos: El estudio analiza retrospectivamente 90 pacientes con gangrena de Fournier tratados en nuestra institución entre 1975 y 2008. Evaluamos la edad media, enfermedades médicas asociadas, el origen de la fascitis necrotizante, el tiempo de evolución y la extensión de la fascitis necrotizante. Los resultados fueron valorados de acuerdo si el paciente sobrevivía o fallecía. En todos los pacientes se realizó desbridamiento quirúrgico extenso y recibieron terapia antibiótica parenteralmente.

Resultados: La tasa de mortalidad fue del 34,4\%. La edad media 63,0 años (rango 33-95), encontramos diferencias estadísticamente significativas entre la edad media de los supervivientes (edad media 59,84 años) y los fallecidos (edad media 70,20 años) ( $\mathrm{p}=0,001)$. Presentaban alguna comorbilidad médica asociada 51 pacientes, la tasa de mortalidad fue mayor en estos pacientes, especialmente en aquellos que sufrían cáncer. Aunque la diabetes mellitus fue la patología más frecuentemente asociada, no se relacionó con un peor pronóstico de modo estadísticamente significativo. El origen de la infección se identificó en 62 pacientes, que presentaron una mayor mortalidad $(\mathrm{p}=0,015)$, la mortalidad cuando existía un origen urológico fue del 50\%. Además, los pacientes que padecían una infección más extensa también mostraron un peor pronóstico.

Conclusiones: La gangrena de Fournier tiene una alta tasa de mortalidad. Para evaluar las posibles variables pronósticas de esta en enfermedad son necesarios estudios con un elevado número de casos. La edad del paciente, la presencia de factores de riesgo, especialmente cáncer, un origen urológico de la infección y la extensión de la enfermedad tienen influencia en el pronóstico de la gangrena de Fournier.
\end{abstract}

Palabras clave: Gangrena de Fournier. Genital masculino. Pronóstico. Tratamiento.

\section{ABSTRACT \\ FOURNIER GANGRENE: EVALUATION OF PROGNOSTIC FACTORS IN 90 PATIENTS}

Introduction: This study evaluates the risk factors and prognostic variables that affect survival of patients with gangrene of Fournier.

Material and methods: The study retrospectively analyzed 90 consecutive patients with gangrene of Fournier treated in our institution between 1975 and 2008. We evaluated the average age, associated systemic diseases, and the source, time of evolution and extent of necrotizing fasciitis. The outcomes were assessed according to whether the patient survived or died. All patients had aggressive surgical debridement, and received parenteral antibiotic therapy.

Results: The mortality rate was $34.4 \%$. The mean age was 63.0 years (range 33-95), a statistically significant difference was found between the age of the survivors (median age, 59.84 years) and those who died (median age, 70.20 years) $(\mathrm{p}=0.001)$. Medical comorbidities were identified in 51 patients; the death rate was higher in patients who had any medical disease, especially those who suffered from cancer. Although diabetes mellitus was the most common associated pathology, it was not related to a statistically significant worst prognosis. The source of the infection was identified in 62 patients, who showed a higher mortality $(\mathrm{p}=0.015)$, the mortality rate when a urological source is identified was $50 \%$. Moreover, patients suffering from a more extensive necrotizing infection showed a worst prognosis.

Conclusions: The gangrene of Fournier has a high mortality rate. Large series are required to study prognostic variables of this disease. The patient age, the presence of systemic risk factors, especially cancer, a urological source of infection and the extent of the disease have impact on the prognosis of Fournier's gangrene.

Keywords: Fournier Gangrene. Male genitalia. Prognosis, Treatment. 
$\mathrm{L}^{\mathrm{a}}$ a gangrena de Fournier es una fascitis necrotizante rápidamente progresiva que se origina en la región genital y perineal. Se extiende siguiendo los planos faciales, pudiendo llegar a afectar la región inguinal, los muslos e incluso la pared abdominal $^{1}$. El cuadro infeccioso provoca la trombosis de los vasos sanguíneos subcutáneos, que va a ser la causa de la gangrena de la piel suprayacente ${ }^{2,3}$.

La enfermedad tal y como la entendemos actualmente difiere del cuadro que describió Jean Alfred Fournier en 1883; una grave gangrena genital idiopática con un debut súbito y una rápida extensión que afectaba a hombres previamente $\operatorname{sanos}^{4-6}$. Los casos descritos actualmente presentan un curso más indolente, un debut menos abrupto, y en muchos casos en posible identificar un foco como origen de la infección ${ }^{7}$. Por otro lado, se describe en varones con un rango de edad más amplio, incluyendo casos con edades avanzadas. Además se observa un aumento de la edad media que oscila desde 40,9 años en los casos descritos antes de 1945 hasta una edad media superior a los 60 años en algunas series más recientes ${ }^{4,9}$. Finalmente, la presencia de enfermedades debilitantes, tales como alcoholismo, cáncer y diabetes pueden promover el desarrollo de la infección ${ }^{7,8,10}$.

La gangrena de Fournier es una verdadera urgencia urológica, con una alta tasa de mortalidad pese a la instauración de un tratamiento adecuado ${ }^{3}$. En algunos estudios recientes, ésta se estima en torno al 20 - 35\%1,3,4,9. Se considera que aquellos pacientes de mayor edad y aquellos afectos de alguna enfermedad debilitante, tales como diabetes, alcoholismo o desnutrición tienen un peor pronóstico $^{3,11,12}$. Lo mismo ocurre en aquellos casos con un tiempo de evolución más largo hasta recibir tratamiento, una mayor extensión y los que requieren colostomía $^{3,7}$. Un bajo nivel socioeconómico también ha sido descrito como otro factor predisponente ${ }^{2,13}$.

En este estudio, pretendemos determinar en base a los pacientes tratados en nuestro servicio, si los marcadores de riesgo clásicamente asociados a un peor pronóstico en la gangrena de Fournier, conllevan una mayor mortalidad.

\section{MATERIAL Y MÉTODOS}

Hemos realizado un estudio retrospectivo en el que revisamos los 90 pacientes diagnosticados y tratados de gangrena de Fournier en nuestro centro entre enero de 1975 y julio de 2008.
El diagnóstico de la gangrena de Fournier se realizó en base a la historia clínica y la exploración física. Se realizaron estudios radiográficos en casos seleccionados para diagnóstico diferencial y determinar la extensión (Fig. 1). Se excluyeron del estudio aquellos pacientes con hernias incarceradas, hidroceles infectados y abscesos escrotales, perirrectales o isquiorrectales que no asociaban infección necrotizante.

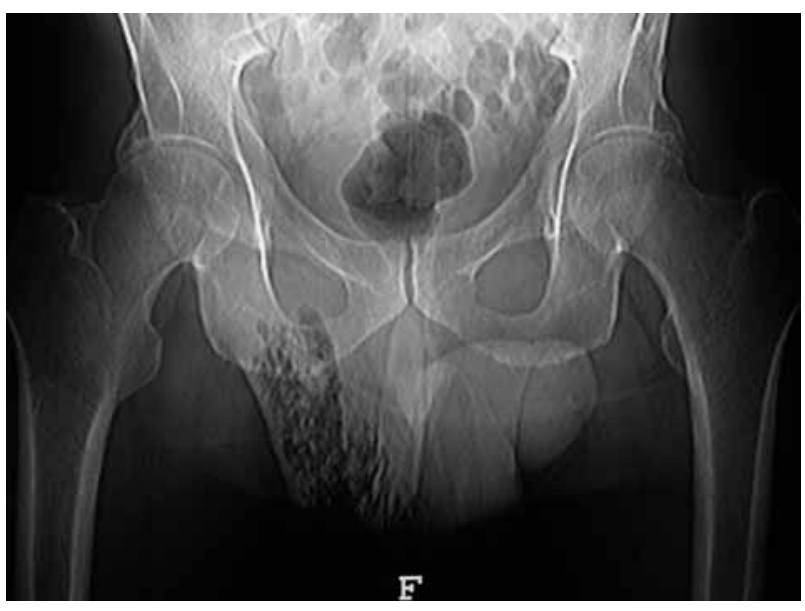

FIGURA 1. Imagen radiográfica en paciente con gangrena de Fournier que pone de manifiesto la presencia de gas.

Las variables pronósticas analizadas fueron la edad del paciente, la presencia de factores predisponentes asociados, entre los que incluimos diabetes mellitus, tumor, inmunodepresión y alcoholismo crónico. También se analizó el posible origen de la infección necrotizante, para ello distinguimos entre aquellos casos con un origen urológico, un origen colorrectal o un origen desconocido. Definimos como origen desconocido, los casos en los que no se puede encontrar una clara causa subyacente relacionada con la gangrena de Fournier. Se valoró el tiempo transcurrido desde la aparición de los primeros síntomas hasta recibir atención médica. De acuerdo a los hallazgos quirúrgicos en el desbridamiento inicial, la extensión del área afecta fue cuantificada utilizando una modificación del nomograma de área corporal, que habitualmente se usa para determinar la extensión de las quemaduras. El pene, el escroto y el periné fueron valorados como una extensión del 1\% cada uno, cada fosa isquiorrectal como un $2,5 \%$ y así sucesivamente. Se trata de un modo similar al descrito por Laor y Palmer ${ }^{11,12}$. 
Se realizó desbridamiento quirúrgico, resecando completamente todo el tejido afecto por la infección necrotizante y drenando el absceso. Se resecó fascia o se realizó orquiectomía en los casos en los que se sospechaba que estuvieran afectos. Además se pautó cobertura antibiótica de amplio espectro a la llegada del paciente a urgencias. En el acto quirúrgico se tomaron muestras de la zona purulenta para cultivo de gérmenes aerobios y anaerobios. Tras la cirugía la zona afecta fue cubierta con comprensas impregnadas con suero y agua oxigenada. La herida era curada diariamente, realizándose desbridamiento si era preciso, resecándose el tejido necrótico.

Las variables fueron analizadas en función de si el paciente sobrevivía o fallecía. Se consideraron muertes relacionadas con la enfermedad, aquellas que ocurrieron durante la estancia hospitalaria, directa o indirectamente, consecuencia del proceso infeccioso. Para el análisis estadístico se utilizaron los test Chi cuadrado y $t$ de Student, en función del tipo de variables comparadas. Se estableció como límite de significación estadística un valor de $\mathrm{p}$ menor o igual a 0,05.

\section{RESULTADOS}

De los 90 pacientes evaluados, 31 fallecieron $(34,4 \%)$ y 59 sobrevivieron (65,6\%). La edad media global fue de 63,08 años $\pm 14,0$, con una mediana de 64 años (rango 33-95). Analizando la diferencia entre la edad media entre los pacientes que fallecen (edad media 70,20 $\pm 13,40$; rango 35-95) y los que sobreviven (edad media 59,84 $\pm 13,17$; rango 33-81) encontramos diferencias estadísticamente significativas entre ambos grupos $(p=0,001$, test $t$ de Student).

Analizando la evolución de la edad media de los pacientes tratados en nuestro servicio, apreciamos que la edad media de los casos tratados en los diez primeros años que incluimos en nuestra serie, desde 1975 a 1984, fue de 59,74 años; mientras que en casos tratados en los últimos diez años muestran una edad media de 62,57 años, con una mediana de 67 años.

La presencia de enfermedades debilitantes, tales como diabetes mellitus, alcoholismo o cáncer, es frecuente en los pacientes que desarrollan una gangrena de Fournier. Identificamos alguna de estas comorbilidades en 51 pacientes (56,7\%). En relación con la tasa de mortalidad, 27 de los supervivientes y 24 de los que fallecen tenían al menos alguna de las enfermedades debilitantes previamente reseñadas. La comorbilidad más frecuentemente asociada fue diabetes mellitus, 28 pacientes eran diabéticos $(31,1 \%) ; 15$ sobrevivieron y 13 fallecieron. Refirieron abuso de alcohol 18 pacientes; 11 sobrevivieron y 7 fallecieron. De los 90 pacientes, 21 padecian algún tipo de cáncer en el momento de la aparición de la gangrena de Fournier, 9 de los cuales sobrevivieron y 12 fallecieron. Al realizar la comparación de las tasas de mortalidad en función de la presencia o ausencia de alguna enfermedad debilitante, encontramos mayores tasas de mortalidad en aquellos pacientes que presentan comorbilidades $(\mathrm{p}=0,004)$. Analizando estos factores de riesgo individualizadamente no hallamos diferencias estadísticamente significativas en cuanto a las tasas de mortalidad en relación a la presencia de diabetes mellitus o alcoholismo crónico. Sin embargo, si se obtienen tasas de mortalidad estadísticamente significativas mayores en los pacientes que padecen cáncer $(\mathrm{p}=0,012)$. De los 51 pacientes que presentan comorbilidades, 16 asociaban dos o más de estas enfermedades debilitantes; y 3 de ellos eran diabéticos, referían alcoholismo y estaban diagnosticados de cáncer (Tabla 1).

Tabla 1. Relación entre la supervivencia y la presencia de enfermedades debilitantes en la gangrena de Fournier ( $\mathrm{n}=90$ )

\begin{tabular}{lcccc}
\hline Variable & Supervivientes (n) & Fallecidos (n) & Total (n) & Valor de p \\
\hline Enfermedad debilitante & 27 & 24 & 51 & 0,004 \\
Diabetes mellitus & 15 & 13 & 28 & 0,140 \\
Alcoholismo & 11 & 7 & 18 & 0,431 \\
Cáncer & 9 & 12 & 21 & 0,025 \\
2 Comorbilidades asociadas & 4 & 9 & 13 & 3 \\
3 Comorbilidades asociadas & 3 & 0 & 3 & \\
\hline
\end{tabular}


La presencia de algún factor local desencadenante de la gangrena de Fournier se encontró en 62 pacientes, 35 sobrevivieron y 27 fallecieron. El origen de la infección se relacionó con una patología del sistema genitourinario en 30 pacientes (33,3\%), 15 sobrevivieron y 15 fallecieron. Un origen colorrectal fue descrito en 32 casos (35,5\%), 20 sobrevivieron y 12 fallecieron. En los 28 pacientes restantes no se encontró ninguna causa con desencadenante de la infección necrotizante, de estos pacientes 24 sobrevivieron y 4 fallecieron. Comparando el posible origen del cuadro con el pronóstico, encontramos tasas de mortalidad estadísticamente significativa más altas en aquellos pacientes en los existe un factor desencadenante de la gangrena de Fournier $(\mathrm{p}=0,015)$ (Tabla 2$)$.

La sintomatología referida comúnmente incluía dolor, edema genital y fiebre. La presencia de una placa necrótica se constató en el $61,1 \%$ de los pacientes (Fig. 2). A su llegada a urgencias, 18 pacientes presentaban estado séptico, de los cuales 5 sobrevivieron y 13 fallecieron $(\mathrm{p}=0,001)$. El tiempo medio desde la aparición de los sintomas hasta recibir tratamiento médico fue de 85,68 horas. Sin embargo no encontramos diferencias significativas entre la duración de los síntomas y el pronóstico de la enfermedad $(81,73 \pm 43,0$ horas frente $87,80 \pm$ 58,3 horas; $\mathrm{p}=0,669$ ).

La superficie corporal media afectada por la infección necrotizante en los pacientes que sobrevivieron y en aquellos que fallecieron fueron 3,29 \% y 4,67, respectivamente. Estas diferencias en cuanto a extensión resultaron estadísticamente significativas $(p=0,032)$.

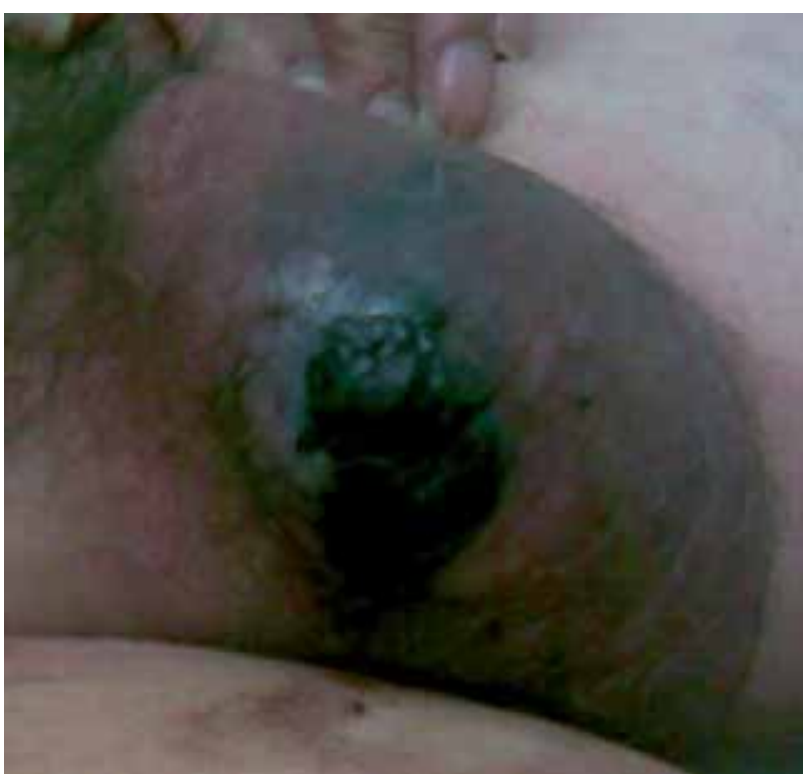

FIGURA 2. Placa necrótica a nivel escrotal.

Tras el diagnóstico de la gangrena de Fournier se realizó un desbridamiento extenso, resecando todo el tejido necrótico y drenando el absceso. Como medidas quirúrgicas auxiliares, practicó una cistostomía suprapúbica en 17 pacientes, 11 sobrevivieron y 6 fallecieron $(\mathrm{p}=0,964)$; fue necesaria orquiectomía en 14 casos, 10 sobrevivieron y 4 fallecieron $(\mathrm{p}=0,592)$. Finalmente, se realizó una colostomía en 31 pacientes, 19 sobrevivieron y 12 fallecieron $(\mathrm{p}=0,575)$.

Los estudios bacteriológicos mostraron flora mixta aerobia y anaerobia en 62 casos $(68,1 \%)$, no crecieron gérmenes anaerobios en 27 casos (30\%) y en un caso los cultivos fueron estériles. La bacterias

Tabla 2. Relación entre la supervivencia y la presencia o ausencia de un origen como causa de la gangrena de Fournier $(\mathrm{n}=90)$

\begin{tabular}{lccc}
\hline Origen de la infección & Supervivientes (n) & Fallecidos (n) & Total (n) \\
\hline Urogenital & 15 & 15 & 30 \\
$\quad$ Prostatismo moderado & 3 & 4 & 7 \\
Sondaje vesical & 2 & 4 & 6 \\
Neoplasia urológica & 2 & 4 & 6 \\
Infección urinaria & 4 & 2 & 6 \\
Cirugía (fimosis, vasectomía) & 2 & 0 & 2 \\
Relación sexual & 1 & 0 & 1 \\
Estenosis uretra & 0 & 1 & 1 \\
Trasplante renal & 1 & 0 & 1 \\
Colorrectal & 20 & 12 & 32 \\
Idiopático & 24 & 4 & 28 \\
\hline
\end{tabular}


aisladas más frecuentemente en los cultivos fueron por este orden Escherichia coli, Bacteroides spp., Proteus spp., Enterococcus. En un caso se aisló Candida albicans en los cultivos tomados durante el acto quirúrgico de la zona purulenta, este paciente era diabético y sufría cáncer (Tabla 3).

Tabla 3. Análisis microbiológico en la Gangrena de Fournier

\begin{tabular}{lc}
\hline Microorganismo & Prevalencia (\%) \\
\hline Escherichia coli & $44,7 \%$ \\
Bacteroides & $31,9 \%$ \\
Proteus & $19,1 \%$ \\
Enterococos & $17,0 \%$ \\
Streptococcus & $17,0 \%$ \\
Staphylococcus & $14,9 \%$ \\
Otras Enterobacterias & $12,8 \%$ \\
Klebsiella & $10,6 \%$ \\
Pseudomona & $8,5 \%$ \\
Clostridium & $6,4 \%$ \\
Fusobacterium & $2,1 \%$ \\
\hline
\end{tabular}

\section{DISCUSIÓN}

Desde que Jean Alfred Fournier describiera la enfermedad que lleva su nombre, se han publicado numerosas series evaluando esta patología. Cuando revisamos los casos publicados observamos un aumento progresivo en la edad media de los pacientes que padecen esta fascitis necrotizante ${ }^{8,14}$. La edad media de los casos publicados antes de 1945 fue 40,9 años ${ }^{2,7}$. Sin embargo, la edad media de series publicadas recientemente oscila en torno a los $60^{4,9,15}$. La edad media de los pacientes que incluye nuestro estudio fue 63,08 años; unos valores acordes a los publicados actualmente. Como hemos realizado una revisión que abarca un periodo de más de treinta años, podemos comprobar la evolución de la edad media de los pacientes tratados, observándose un aumento desde los 59,7 años de media de los pacientes tratados entre 1975 a 1984, a los 62,57 años de media en los tratados en los últimos diez años. Algunos estudios publicados recientemente no demuestran diferencias significativas entre la edad de los pacientes que sobreviven y los falleci$\operatorname{dos}^{4,9}$. Sin embargo estudios clásicos de esta patología, como los publicados por Clayton et al. ${ }^{16} \mathrm{y}$ por
Laor et al. ${ }^{11}$ observan que los pacientes que sobreviven son significativamente más jóvenes que los fallecidos. Es necesario mencionar que estos últimos estudios incluyen un mayor número de pacientes. Como la gangrena de Fournier es una enfermedad poco frecuente, estudios con un número elevado de pacientes son necesarios para valorar las variables que influyen en el pronóstico, lo que supone series en engloban largos periodos. Muchas de las series que se han publicado valorando esta patología tienen un pequeño número de pacientes lo que dificulta la obtención de significación estadística. Revisando los 90 pacientes tratados en nuestro servicio en un periodo de 33 años, encontramos que los pacientes que sobreviven son significativamente más jóvenes que aquellos que fallecen. Por lo tanto, la edad tiene influencia en el pronóstico, este hecho tiene una doble importancia ya cada vez es más frecuente tratar a pacientes con edades avanzadas.

Se ha asociado el desarrollo de la gangrena de Fournier con el padecimiento de ciertas enfermedades debilitantes, tales como diabetes mellitus, alcoholismo crónico y cáncer ${ }^{7,8,10,17}$. En nuestra serie observamos que 24 de los 31 fallecidos presentaban alguna de estas comorbilidades; referidas en 27 de los 59 supervivientes. Los pacientes que sufrían alguna enfermedad debilitante presentan un pronóstico significativamente peor. La diabetes mellitus es el factor de riesgo más frecuentemente asociado al padecimiento de gangrena de Fournier ${ }^{3,15}$. Podemos afirmar que la prevalencia de diabetes mellitus en los pacientes con gangrena de Fournier es mayor que en la población general ${ }^{18}$. Sin embargo, cuando revisamos la literatura, encontramos cierta controversia a la hora de discernir si la coexistencia de diabetes mellitus influye el pronóstico $^{19}$. Aunque existen numerosos estudios que muestran que el padecimiento de diabetes mellitus incrementa el riesgo de desarrollar una gangrena de Fournier, pero no encuentran diferencias significativas en cuanto al pronóstico ${ }^{4,9,17}$. De acuerdo con estos resultados, Nisbet et al. muestran que los pacientes diabéticos requieren un mayor número de desbridamientos quirúrgicos con resecciones de tejido más extensas ${ }^{17}$. En nuestro estudio, aunque encontramos una tasa de mortalidad mayor en los pacientes diabéticos, la diferencia no es estadísticamente significativa. La cosa es diferente a la hora de evaluar otro factor predisponente como es el padeci- 
miento de cáncer, estos pacientes si presentan un peor pronóstico. En función de estos datos, se puede concluir que los pacientes que padecen alguna enfermedad maligna presentan un peor pronóstico, sin embargo son necesarios más estudios con un número de pacientes elevado para evaluar detalladamente la influencia del padecimiento de diabetes mellitus en el pronóstico y evolución de la gangrena de Fournier.

Como origen del proceso infeccioso, es posible encontrar una causa urogenital, colorrectal o cutánea. El origen colorrectal suele ser el más frecuentemente descrito, un 35,5\% en nuestra serie; y con frecuencia se ha descrito asociado a un peor pronóstico ${ }^{7,20}$. Desde el punto de vista urológico, se describen muy diversos factores como posibles desencadenantes de una gangrena de Fournier, tales como el padecimiento de un tumor vesical, pacientes portadores de sonda vesical, todo tipo de infecciones genitourinarias, la presencia de una estenosis uretral ${ }^{2,16,20,21}$, e incluso se han descrito casos en relación a una vasectomía o una circuncisión los días previos ${ }^{8,13,22}$. Nuestra serie con un porcentaje de casos con origen urológico $(33,3 \%)$ muy similar a los casos con origen digestivo, incluye un caso asociado a la realización de una vasectomía, uno se asocia a una circuncisión en los días previos y otro en relación a un traumatismo en una relación sexual. Aunque Paty et al. determinaron que en un 95\% de los pacientes es posible hallar un posible origen $^{7}$, nuestra tasa de casos idiopáticos supone un $31,1 \%$, similar a los obtenidos en otros estudios más recientes ${ }^{14}$. Aunque más que en el porcentaje de casos idiopáticos, quisiéramos llamar la atención, en el peor pronóstico que hemos encontrado cuando existe un origen subyacente como causa de la gangrena, especialmente cuando el origen es urológico, con una mortalidad del 50\%.

A pesar de que la gangrena de Fournier es una fascitis necrotizante rápidamente progresiva que requiere la instauración de un tratamiento lo más precozmente posible, el tiempo que ha trascurrido desde la aparición de los primeros sintomas oscila de dos a siete días ${ }^{1,2,4,7}$, resultados similares a los que arroja nuestro estudio. Para valorar estos tiempos de evolución, es preciso mencionar que síntomas leves como malestar genital, pueden preceder en varios días a la aparición la clásica placa necrótica y el desarrollo de la gangrena ${ }^{7}$. Los principios para manejar esta enfermedad no han cambiado con el paso de los años ${ }^{23}$. El tratamiento se basa en dos aspectos fundamentales, en primer lugar son necesarias las medidas de soporte y reanimación básica del paciente ${ }^{2,7}$ junto con la instauración de un tratamiento antibiótico de amplio espectro, en segundo lugar es preciso un tratamiento quirúrgico inmediato realizando una resección amplia de todos los tejidos necrosados hasta llegar a tejido sano ${ }^{8,14}$, este amplio abordaje quirúrgico tiene una repercusión positiva en la supervivencia ${ }^{4}$. No se trata, por lo tanto, de un simple desbridamiento de los tejidos, sino que la resección debe incluir aquellas zonas edematosas y con crepitación que indican el frente de propagación de la infección. Para Tuncel et al. la superficie corporal afectada es uno de los factores predictivos de mortalidad más importantes en los pacientes con gangrena de Fournier ${ }^{9}$. Por otro lado, aunque Palmer et al. afirmaron que la supervivencia no era directamente proporcional al área afecta y también sugirieron que aquellos pacientes con una extensión limitada de la enfermedad tienen más probabilidades de sobrevivir ${ }^{12}$. En función de los resultados que obtenemos en nuestra serie podemos concluir que la extensión de la gangrena tiene repercusión en la mortalidad, observándose una mayor superficie corporal afecta por la gangrena en los pacientes fallecidos. Aunque a la hora de comparar el tiempo de evolución del proceso y la mortalidad no encontramos diferencias significativas, pensamos que como la extensión de la gangrena y el tiempo de evolución son variables relacionadas, es lógico pensar que un mayor tiempo de evolución debería asociarse a una extensión más amplia.

Desde el punto de vista microbiológico, la gangrena de Fournier está causada por la acción sinérgica de microorganismos aeróbicos y anaeróbicos, aunque con frecuencia no se aíslan anaerobios en los cultivos $^{2,23}$, un $31 \%$ de los casos en nuestra serie. En los cultivos tomados para el análisis microbiológico generalmente crecieron las mismas bacterias que habitualmente constituyen la flora uretral, rectal y cutánea ${ }^{2,3,8,24}$. Nuestros cultivos microbiológicos generalmente mostraron una flora polimicrobiana. Entre los gérmenes aerobios, los aislados con más frecuencia fueron Escherichia coli, Proteus y Enterococcus; mientras que los Bacteroides fueron los microorganismos anaerobios aislados con más frecuencia. Algunos autores sugie- 
ren que cada vez es más frecuente encontrar gérmenes atípicos ${ }^{23}$. Se han publicado casos en los que se han cultivados organismos tales como Candida y sparganum $^{25,26}$. Nuestra serie incluye un paciente en el creció una Candida en los cultivos microbiológicos. En función de estos resultados, se recomienda administrar una terapia antimicrobiana con actividad frente a cocos grampositivos, bacilos gramnegativos $\mathrm{y}$ anaerobios ${ }^{8,27}$. Estudios publicados recientemente aconsejan la administración de cefalosporinas de tercera generación y metronidazol, pudiéndose añadir gentamicina ${ }^{9,20,23}$; una terapia similar a la que utilizamos nosotros.

\section{CONCLUSIÓN}

La gangrena de Fournier tiene una alta tasa de mortalidad pese a un tratamiento agresivo con tratamiento antibiótico intravenoso y un amplio abordaje quirúrgico resecando la zona afecta. La población de edad más avanzada y aquella que sufre alguna enfermedad debilitante, cualidades que suelen ir asociadas, tienen un peor pronóstico. Además cada vez es más frecuente tratar pacientes de mayor edad. Las tasas de mortalidad más altas son especialmente destacables en los pacientes que padecen cáncer o presentan un origen urológico de la infección necrotizante. Se trata de una enfermedad que requiere un diagnostico precoz ya que una mayor extensión de la enfermedad también se asocia a un peor pronóstico.

\section{REFERENCIAS}

1. Dahm P, Roland FH, Vaslef SN, Moon RE, Price DT, Georgiade GS, et al. Outcome analysis in patients with primary necrotizing fasciitis of the male genitalia. Urology. 2000;56(1):31-36.

2. Smith GL, Bunker CB, Dinneen MD. Fournier's gangrene. Br J Urol. 1998;81(3):347-355.

3. Jeong HJ, Park SC, Seo IY, Rim JS. Prognostic factors in Fournier gangrene. Int J Urol. 2005;12(12):1041-1044.

4. Yeniyol CO, Suelozgen T, Arslan M, Aayder AR. Fournier's gangrene: experience with 25 patients and use of Fournier's gangrene severity index score. Urology. 2004;64(2):218-222.

5. Fournier JA. Gangrene foudroyante de la verge. Sem Med. 1883;3:345-347.

6. Fariña LA, Zungri ER. Gangrena de Fournier y otras infecciones necrotizantes. Arch Esp Urol. 2001;54(10):1061-1065.

7. Paty R, Smith $\mathrm{AD}$. Gangrene and Fournier's gangrene. Urol Clin North Am. 1992;19(1):149-162.

8. Rodríguez Alonso A, Pérez García MD, Núñez López A, Ojea Calvo A, Alonso Rodrigo A, Rodríguez Iglesias B, et al. Gangrena de Fournier: aspectos anátomo-clínicos en el adulto y en el niño. Actualización terapéutica. Actas Urol Esp. 2000;24(4):294-306.

9. Tuncel A, Aydin O, Tekdogan U, Nalcacioglu V, Capar Y, Atan A. Fournier's Gangrene: Three years of experience with 20 patients and validity of the Fournier's Gangrene severity index score. Eur Urol. 2006;50(4):838-843.
10. Basoglu M, Ozbey I, Atamanalp SS, Yildirgan MI, Aydinli B, Polat O, et al. Management of Fournier's Gangrene: Review of 45 Cases. Surg Today. 2007;37(7):558-563.

11. Laor E, Palmer LS, Tolia BM, Reid RE, Winter HI. Outcome prediction in patients with Fournier's gangrene. J Urol. 1995;154 (1):89-92.

12. Palmer LS, Winter HI, Tolia BM, Reid RE, Laor E. The limited impact of involved surface area and surgical débridement on survival in Fournier's gangrene. Br J Urol. 1995;76(2):208-212.

13. Eke N. Fournier's gangrene: a review of 1726 cases. Br J Surg. 2000;87(6):718-728.

14. Irazu JC, de Miceu S, Salas J, Katz ON, Echeguren ES, Blundo OA. Gangrena de Fournier. Nuestra experiencia clínica, etiopatogenia y tratamiento. Actas Urol Esp. 1999;23(9):78-83.

15. Xeropotamos NS, Nousias VE, Kappas AM. Fournier's Gangrene: Diagnostic approach and therapeutic challenge. Eur J Surg. 2002;168(2):91-95.

16. Clayton MD, Fowler JE Jr, Sharifi R, Pearl RK. Causes, presentation and survival of fiftyseven patients with necrotizing fasciitis of the male genitalia. Surg Gynecol Obstet. 1990;170 (1):49-55.

17. Nisbet AA, Thompson IM. Impact of diabetes mellitus on the presentation and outcomes of Fournier's gangrene. Urology. 2002;60(5):775-779.

18. Ruiz Ramos M, Escolar Pujolan A, Mayoral Sánchez E, Corral San Laureano F, Fernández Fernández I. La diabetes mellitus en España: mortalidad, prevalencia, incidencia, costes económicos y desigualdades. Gac Sanit. 2006;20(Supl 1):15-24.

19. Morpurgo E, Galandiuk S. Fournier's gangrene. Surg Clin North Am. 2002;82(6):1213-24.

20. Quatan N, Kirby RS. Improving outcomes in Fournier's gangrene. BJU Int. 2004;93(6):191-192.

21. Gómez Pérez L, Delgado Oliva FJ, Gimeno Argente V, Arlandis Guzmán S, Arce Casado B, Jiménez Cruz FJ. Gangrena de Fournier con afectación uretral: reepitelización uretral con tratamiento conservador. Actas Urol Esp. 2006;30(1):101.

22. De Diego Rodríguez E, Correas Gómez MA, Martín García B, Hernández Rodríguez R, Portillo Martín JA, Gutiérrez Baños JL, et al. Gangrena de Fournier postvasectomía. Arch Esp Urol. 2000;53(3):275-278.

23. Verit A, Verit FF. Fournier's gangrene: The development of a classical pathology. BJU Int. 2007; 100(6):1218-20.

24. Ersay A, Yilmaz G, Akgun Y, Celik Y. Factors affecting mortality of Fournier's Gangrene: review of 70 patients. ANZ J Surg. 2007;77(1-2):43-48.

25. Johnin K, Nakatoh M, Kadowaki T, Kushima M, Koizumi S, Okada Y. Fournier's gangrene caused by Candida species as the primary organism. Urology. 2000;56(1):153XIII-153XV.

26. Jeong HJ. Fournier's gangrene associated with sparganosis in the scrotum. Urology. 2004;63(1):176XXI-176XXII.

27. Planelles Gómez J, Vergés Prósper A, Rubio Tortosa I, Beltrán Armada JR, Carrascosa Lloret V, San Juan de Laorden C. Gangrena de Fournier. Arch Esp Urol. 2006;59(8):767-71.

Correspondencia autor: Dr. J. Medina Polo Servicio de Urología

Hospital Universitario 12 de Octubre

Avda. de Andalucía, s/n - 28041 Madrid

Tel.: 913908121

E-mail autor: josemedinapolo@telefonica.net Información artículo: Gangrena de Fournier Trabajo recibido: septiembre 2008

Trabajo aceptado: octubre 2008 